http://jmscr.igmpublication.org/home/

ISSN (e)-2347-176x ISSN (p) 2455-0450

crossref DOI: https://dx.doi.org/10.18535/jmscr/v7i11.143

Journal Of Medical Science And Clinical Research

Original Article

\title{
Dosimetric evaluation of bladder and rectal radiation dose in Ca Cervix patients using CT planning with Iridium 192, Cobalt 60 in HDR Brachytherapy-Single Institutional Experience
}

\author{
Authors \\ Dr N. V. Kalaiyarasi DCH MDRT, Professor and HOD, \\ Dr S Madhumathi* DMRT, MDRT \\ *Corresponding Author \\ Dr Madhumathi.S \\ Senior Assistant Professor, Department of Radiotherapy
}

Source(s) of support: Department of Radiation Oncology \& Radiation Physics, MMC/RCC, Kancheepuram

\begin{abstract}
Context: Dosimetric evaluation of bladder and rectal Radiation dose in Ca Cervix patients using CT planning using Iridium 192 and Cobalt 60 in HDR Brachytherapy-Single Institutional Experience

Aim: To compare the doses of Bladder and Rectal radiation dose in advanced cases of Carcinoma Cervix, using CT simulation iridium 192, Cobalt-60 as source in HDR Brachytherapy, two arm study.

Material and Methods: This is a Comparative descriptive study in single institution

done in 60 eligible patients of age 30-60 years who had Squamous Cell Carcinoma of cervix completed EBRT and slated for Brachytherapy of all stages upto III B during the period of, January 2010 to August 2010 - Arm A, January 2016 to June 2016 - Arm B. All patients were treated with conventional EBRT 50 Gy in 2 Gy/\# for 5 days a week/25\# followed by 8 Gy in 2\# for ICA application, using iridium 192 (Arm A) Cobalt 60 (Arm B) Treatment completed within maximum period of $10-12$ weeks.

Intracavitary Brachytherapy and Dosimetry: Orthogonal X-ray and CT simulation was taken and Point $A$ and point B Bladder and rectal were studied after treatment was carried out using iridium 192 as source Arm A. CT simulation of $3 \mathrm{~mm}$ thickness was obtained, $5 \mathrm{~cm}$ from top of uterine tandem superiorly and to the level of $2 \mathrm{~cm}$ below vaginal introitus inferiorly (From Barnard institute of Radiology). HRCTV, Rectum and Bladder were contoured. Treatment planning was carried out using HDR Plus treatment planning system. Point A and point B, Bladder and rectal doses were studied after treatment was carried out using cobalt-60 HDR remote after loading machine for Arm B.

Results: Out of the 60 patients the average dose received by Point A, Point B, Bladder and Rectal points was within tolerable limits. Early side effect of Organ at Risk, Bladder and Rectum was calculated with BED which is acceptable. Arm A

Average doses received by Poin A - 86.7 (LDR equivalent) Bladder-D2 Volume Rectal D2 Volume

Arm B

Average dose received by Point A - 74.1 Gy, Point B - 56.8 Gy, Bladder-D2 Volume : 7.5 Gy, Rectum D2 Volume: 6.7 Gy. Response assessment was done immediately and it was complete response in 98\% with minimal residual disease in a single patient. Toxicity assessment of Bladder and Rectum were done. Only Grade 1 and Grade 2 reactions were present in both. Patients are under follow up study to assess disease free survival rate and late complications of bladder and rectum.

Conclusion: CO 60 HDR Brachytherapy is not producing bladder and rectal complications and equally shows results on par with Iridium. With long half life it is economical to run CO6O HDR Brachytherapy in treating Carcinoma Cervix.
\end{abstract}




\section{Introduction}

Carcinoma of the uterine cervix $(\mathrm{Ca} \mathrm{Cx})$ is the fifth most common cancer worldwide, the second most common cancer in women and the most common cause of death in cancer patients in the developing countries. ${ }^{1}$ The annual incidence of $\mathrm{Ca}$ $\mathrm{Cx}$ is approximately 510,000 new cases worldwide, with approximately 288,000 deaths. ${ }^{2}$ The $\mathrm{Ca} \mathrm{Cx}$ occurs in the reproductive period of a woman's life. The incidence rises in 30-34 years of age and peaks at 55-65 years, with a median age of 38 years (age 21-67 years).Usually, the $\mathrm{Ca}$ $\mathrm{Cx}$ patients are treated with weekly chemotherapy concurrent with external beam radiotherapy (EBRT) for 50 Gray in 25 fractions (50 Gy/25\#) at the rate of 2 Gy/\#3followed by biological equivalent dose of 30-35 Gy by3\# of 7-8.5 Gy or 2\# of 9 Gy by high dose rate (HDR) brachytherapy. Brachytherapy has an inverse square law as the most dominant physical effect, whereby radiation dose is inversely proportional to the square of the distance from the source. In practical terms, this allows for a very high dose to the tumour with relative sparing of the surrounding normal structures, which is the only demonstrated method of providing the high dose required to control $\mathrm{Ca} \mathrm{Cx}$ (>80 Gy) without exceeding the dose tolerance of the bladder and rectum. ${ }^{4}$ Ir-192 HDR brachytherapy units has been used for a long time. Co-60 HDR unit has been introduced in past few years and is gaining importance owing to its long half-life. Co-60with enhanced activity allows miniaturized sources that are equivalent to conventional Ir-192 sources (as shown in Table 1) with the same shape and diameter of applicators and similar application techniques. ${ }^{6}$ The Co-60 and Ir-192 sources decay with different half-lives (T1/2), the Co-60 source replacement is done after five years while Ir-192 source is replaced at every four months and both sources have different initial conditions. The variation in treatment time with time for the two sources is as shown in Fig. ${ }^{1.7}$ Long half life of Co60 provides relaxation in terms of repeated source replacement, documentation and administrative workload, specially related to competed authority, and thus, above all, it is economical as compared to Ir-192 HDR brachytherapy unit. Although, due to higher energy of Co-60 (1.25 MeV) than that of Ir-192 $(0.38 \mathrm{MeV})$, the brachytherapy room with a larger wall thickness is required which increases the initial setup cost of Co-60 HDR brachytherapy unit. ${ }^{8}$ Co-60 HDR brachytherapy offers a clinical outcome equivalent toIr-192 HDR brachytherapy. ${ }^{6,9}$. The physical properties of Co60 and Ir-192 HDR brachytherapy sources are mentioned in Table 1. With the advent of better imaging modalities, radiation technology and its successful implementation in external beam radiation therapy (3D CRT, IMRT, IGRT etc.,) is a success story today. There are many ways to reduce the bladder and rectal complications. High precision radiotherapy techniques like 3DCRT, IMRT are evolving to deliver high radiation dose to the tumour and sparing the critical normal structures. In India, locally advanced cervical cancer being a major problem, appropriate, adequate and quality treatment to all patients is the key to success. In India, there has been a rapid rise in corporate and steady improvement in radiation facilities in government sectors. Most of the study should be done by all high volume centres to incorporate some form of image based approach both for teletherapy and brachytherapy for cervical cancers from India facilities have CT scanners / simulators for RT Planning. There is an urgent to generate robost data on CT or MR 3D Image Based Brachytherapy Planning and suitable evidence through clinical studies to resolve the issues further in optimizing treatment for cervical cancers.

Table1: Physical Properties of Ir-192 and Co-60 radioisotopes. ${ }^{5,6,10}$

\begin{tabular}{|l|c|c|}
\hline Properties & Cobalt-60 & Iridium -192 \\
\hline Half life & 5.26 years & 73.8 days \\
\hline Photon energy $(\mathrm{MeV})$ & $1.25($ mean $)$ & $0.38($ mean $)$ \\
\hline Half value layer(mm lead) & 11 & 2.5 \\
\hline Exposure rate constant $\left(\mathrm{R}-\mathrm{cm}^{2} / \mathrm{mGi}-\mathrm{h}\right)$ & 13.07 & 4.69 \\
\hline Maximum specific activity $(\mathrm{GBq} / \mathrm{mg})$ & 41.91 & 340.98 \\
\hline Inner length & $3.5 \mathrm{~mm}$ & $3.6 \mathrm{~mm}$ \\
\hline Outer length & $5 \mathrm{~mm}$ & $4.5 \mathrm{~mm}$ \\
\hline Inner diameter & $0.5 \mathrm{~mm}$ & $0.65 \mathrm{~mm}$ \\
\hline Outer diameter & $1 \mathrm{~mm}$ & $0.9 \mathrm{~mm}$ \\
\hline
\end{tabular}




\section{Aim}

To compare the doses of Bladder and Rectal radiation dose in advanced cases of Carcinoma Cervix, using CT simulation with iridium 192, Cobalt-60 as source in HDR Brachytherapy, two arm study.

\section{Subjects and Methods}

Approval from institutional ethical committee was taken and consent from all patients was received after explaining the pros and cons of the procedure

Study Period: ARM A - June 2010- August 2010, ARM B- January 2016 - June 2016

Study Design: Comparative descriptive study

Case Selection: Biopsy proven squamous cell Carcinoma Cervix cases of stage IIB - IIIB who had completed EBRT and slated for HDR brachytherapy.

Number of Patients: 30 for both ARM A and ARM B

\section{Inclusion Criteria}

$>$ All eligible biopsy proven squamous cell carcinoma cervix patients, who had completed EBRT and slated for HDR Brachytherapy.

Stage II B - III B

$>$ Age: 30 - 60 years.

$>$ Performance Status: ECOG 0-1 histological proof from primary lesion.

$>$ Informed consent to be taken.

$>$ Patient should be fit for anesthesia.

\section{Exclusion Criteria}

$>$ Age $>60$ years.

$>$ ECOG more than 2.

$>$ Patients not fit for anesthesia.

$>$ Patients with stage IV disease.

$>$ Histology other than squamous cell carcinoma.

$>$ History of prior radiotherapy / chemotherapy to Ca cervix.

\section{Pre-Treatment Evaluation}

History.
> Physical examination with emphasis on gynecological examination.

$>$ Biopsy.

$>$ LAB studies (CBE, LFT, RFT, VDRL, and HIV).

$>$ ECG.

$>$ Radiographic studies,

$>$ Chest X-ray.

$>$ USG abdomen and pelvis to assess primary disease local extension, nodal status and visceral metastasis.

$>$ CT Pelvis.

$>$ Cystoscopy.

$>$ Per speculum examination and by passing uterine sound to assess whether the patient is fit for intra cavity application.

\section{Treatment Protocol}

Conventional EBRT - 50 Gy in 200c Gy per fraction 5 days a week 25 fraction. EBRT equipment : Co-60 Phoenix

Chemotherapy: concurrent chemo with weekly inj Cisplarin $40 \mathrm{mg} / \mathrm{m}^{2}$

Treatment portals: AP and PA portals daily/AP, PA and opposing lateral portals

SIMULATION AND TREATMENT DELIVERY

\section{ICA Protocol}

After teletherapy the patients were assessed for intracavitary application. Those found fit were subjected to high dose rate (HDR) Brachytherapy.

\section{HDR Brachytherapy Protocol ARM A}

Technique Remote after loading with Iridium-192 source

Machine HDR-micro Selectron Activity 2-10 Ci

Intracavitary applicator Modified Fletcher Suit applicator.

No. of \#s TWO (1\# -1 week after EBRT, 2\# -1 week after1\#)

Dose delivered to Point A 800cGy /\# -2\#

Total Dose to point A 86.7Gy (LDR equivalent).LQED $81 \mathrm{~Gy}$

ARM B

Technique Remote after loading with Cobalt-60 source 
Intracavitary applicator provided with Cobalt 60 HDR .

No. of \#s TWO (1\# -1 week after EBRT, 2\# -1 week after1\#)

Dose delivered to HRCTV, Point A 800cGy /\# -2\#

Total Dose to point A 74.1Gy (LDR equivalent).BED 88.8

\section{Procedure}

\section{ARM A}

Under I.V. Sedation patient in routine way Intracavitory Application done. Orthogonal Xrays $\mathrm{AP}$ and lateral were taken. $\mathrm{CT}$ simulation was done. Subsequently the films were digitized and dose calculations were obtained using Nucletron Plato brachytherapy Treatment Planning System. The dose was prescribed to Point -A.

\section{ARM B}

Under short general anaesthesia patient in routine way Intracavitory Application done. CT Stimulation was taken. Subsequently the films were digitized and dose calculations were obtained using brachytherapy Treatment Planning System. The dose was prescribed to Point -A.

\section{Dose Specifications}

\section{ARM A}

Point A and Point B were defined according to Manchester system of dose definition. The bladder and rectal reference points defined by ICRU 38 were used to calculate the bladder and rectal point dose .Four additional rectal points were taken 1 $\mathrm{cm}$ above and below the usual rectal reference points to get better idea of dose received by rectum.

\section{ARM B}

CT images of $3 \mathrm{~mm}$ slice thickness were taken. The CT images were imported on treatment planning system (TPS) HDR Plus. Since the HDR brachytherapy procedures were CT based, the target delineation and planning for all the patients was done as per the American Brachytherapy Society (ABS) guide-lines. High risk clinical target volume (HRCTV) included the entire uterine cervix and any parametrial extension. Organs at risk (OARs) such as the bladder, rectum and sigmoid were also delineated. The planning for $8 \mathrm{~Gy} / \#$ was done following the ABS guidelines. The three channel applicators viz. left lateral, central and right lateral applicators were selected and aligned with the respective applicators seen on CT images. The point ' $A$ ' and point ' $B$ ' were marked by the Manchester system. Step size of $3 \mathrm{~mm}$ was selected and required length of each applicator was activated depending upon the case. The $100 \%$ dose was calculated on point ' $A$ ' and then the $100 \%$ isodose line was adjusted using the available tools on TPS in such a way that HRCTV was covered at least by $90 \%$ of the isodose line. The $100 \%$ isodose line was adjusted to save the bladder, rectum and to reduce the unwanted dose spillage outside the HRCTV volume. Since the planning was three dimensional imaging based, dose to HRCTV was given importance rather than point ' $A$ ' and ' $B$ '. The doses to target and OARs were analyzed and noted. Fig. 2(a) shows the applicators position and Fig. 2(b) shows the pear shaped dose distribution. The patient was shifted to the brachytherapy room and the source guide tubes were connected to the respective applicators. After checking the connections of guide tubes with HDR unit and applicators, the treatment plan was executed. All the patients were treated with $3 \#$ with one week interval between fractions.

\section{Conventional Dosimetry}

Orthogonal radiographs (anterior-posterior and lateral) were taken on a conventional simulator with radio opaque markers in the applicators. The cervical stopper is identified in orthogonal film and used as origin radiographs were reconstructed and the treatment planning was done using PLATO planning system. Source positions were loaded according to the standard loading pattern in accordance with the Manchester system. Point A was defined on the radiographs as being $2 \mathrm{~cm}$ superior to the cervical stopper (flange) and $2 \mathrm{~cm}$ lateral from the axis of the intrauterine tandem. Bladder and rectal reference points were identified according to ICRU 38 recommendations. 


\section{JMSCR Vol||07||Issue||11||Page 818-829||November}

In addition to the ICRU rectal reference point, four additional rectal points were defined at $1 \mathrm{~cm}$ interval superior and inferior to the ICRU rectal reference point. The dose was prescribed to point A; treatment was carried out using Iridium HDR remote after-loading machine based on the conventional radiograph-based dosimetry.

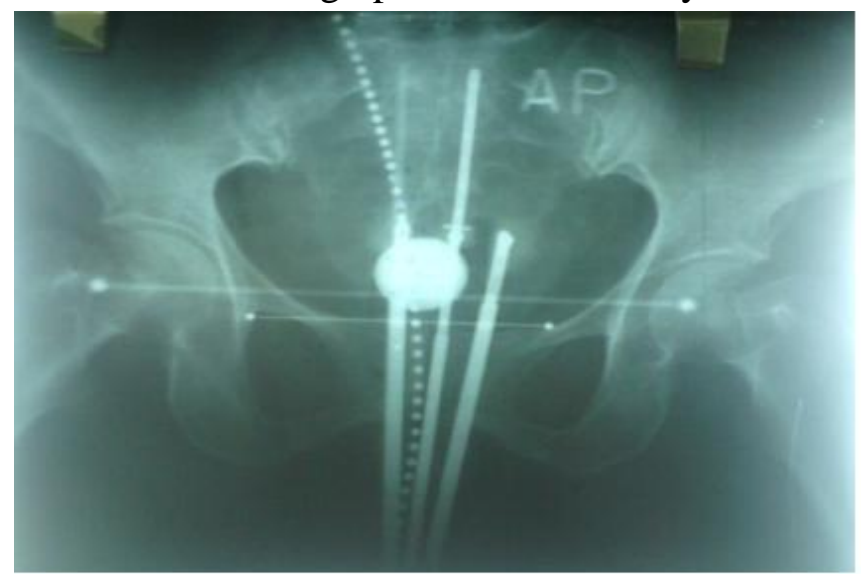

Figure 1 Orthogonal X-Ray Based ICBT - AP Film

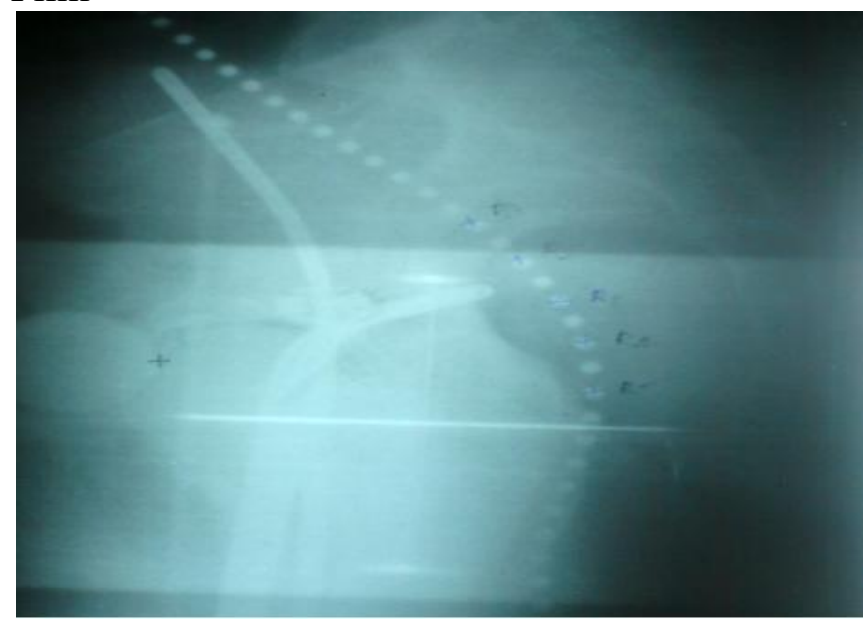

Figure 2 Orthogonal X-Ray Based ICBT Lateral Film

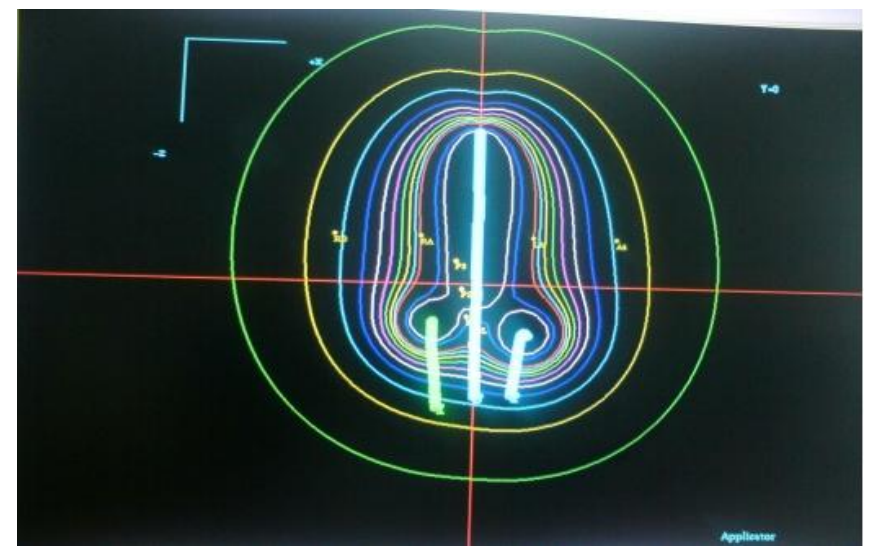

Figure 3 Isodose Distributions in Conventional Planning AP View

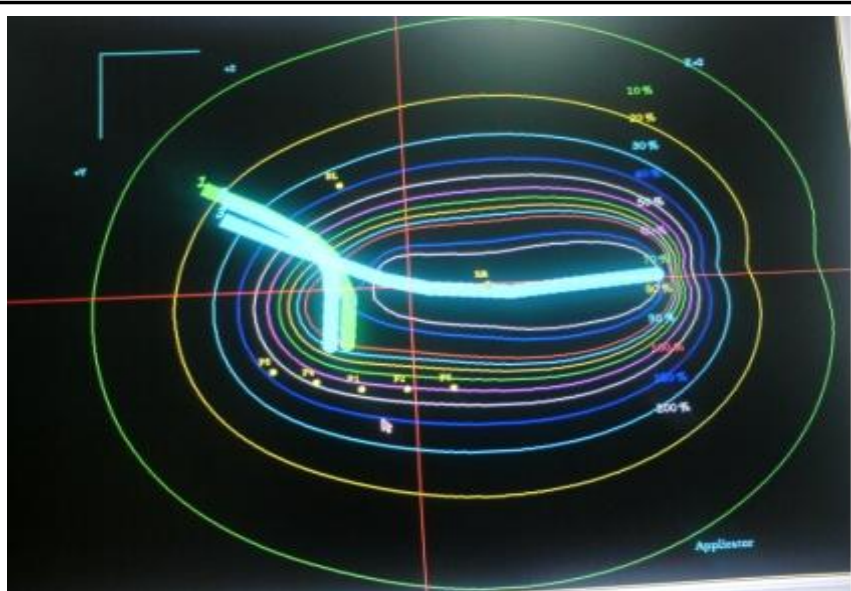

Figure 4 Lateral View

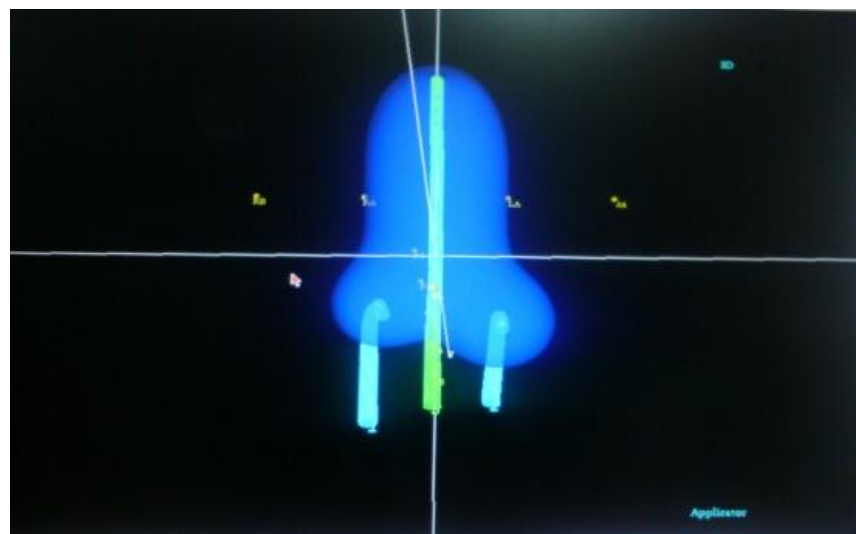

Figure 5 100\% Dose Cloud in 2D Planning

\section{CT-Based ICA-HDR Dosimetry}

All the above intracavitary applications were simultaneously taken up for CT planning. CT scans of 3-mm slice-thickness were obtained, 4 $\mathrm{cm}$ above the tandem superiorly and to the level of anus inferiorly.

Rectum and bladder were delineated. Rectum was contoured from recto-sigmoid junction superiorly till ischial tuberosity inferiorly. The entire bladder was contoured. Treatment planning was carried out using PLATO planning system.

Point A, ICRU rectal and bladder reference points were identified on CT planning. For each application, the corresponding optimized source positions used in radiograph-based planning were duplicated for CT image-based planning .Reconstruction of metal applicators using CT images was difficult due to the presence of artifacts. All bladder and rectal point doses were individually and cumulatively assessed. A statistical analysis was done. The results were obtained. 


\section{JMSCR Vol||07||Issue||11||Page 818-829||November}

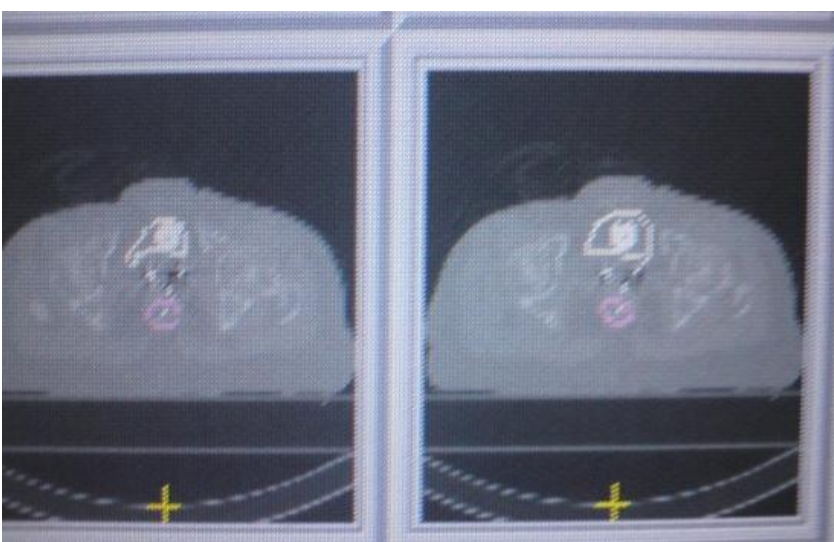

Figure 1 CT Simulation with Bladder and Rectum Contoured

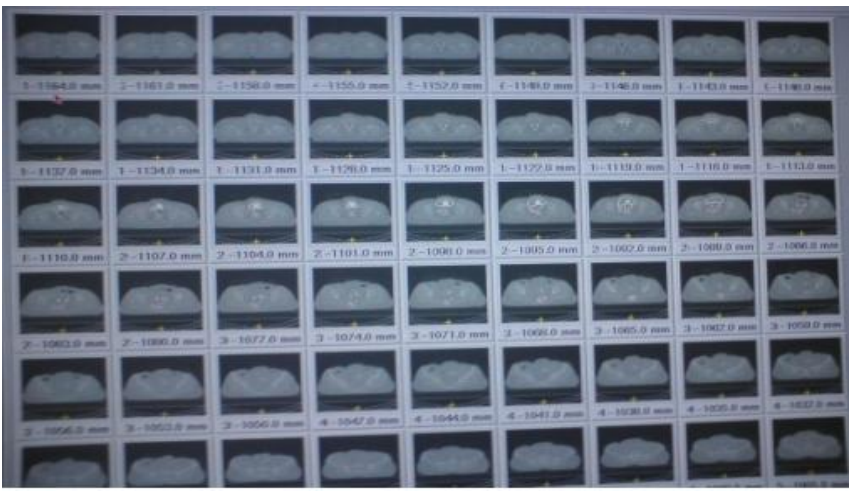

Figure 2 CT Simulation Pictures Axial Cuts

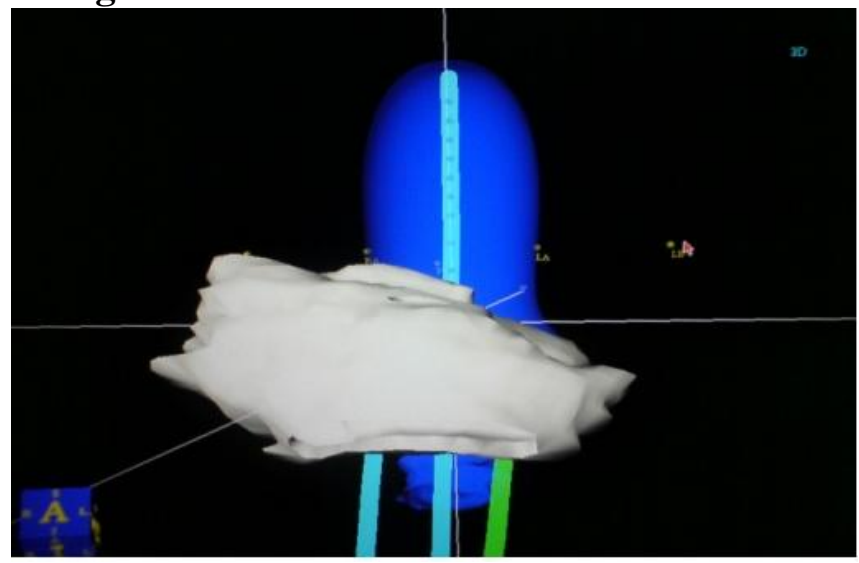

Figure 3 100\% Dose Cloud in 3D Planning

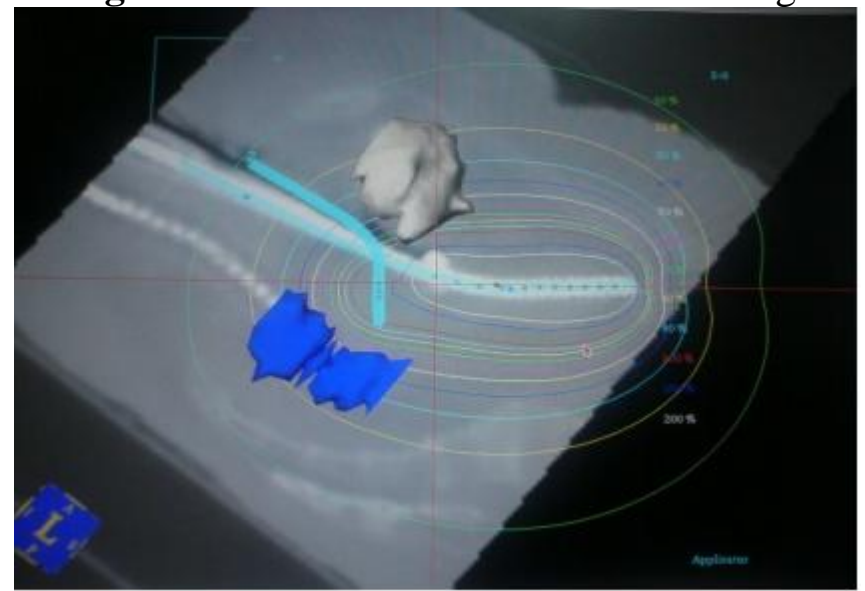

Figure 4 3D Reconstructed Image with Bladder \& Rectum Contoured and Iso Dose Distribution Lateral View

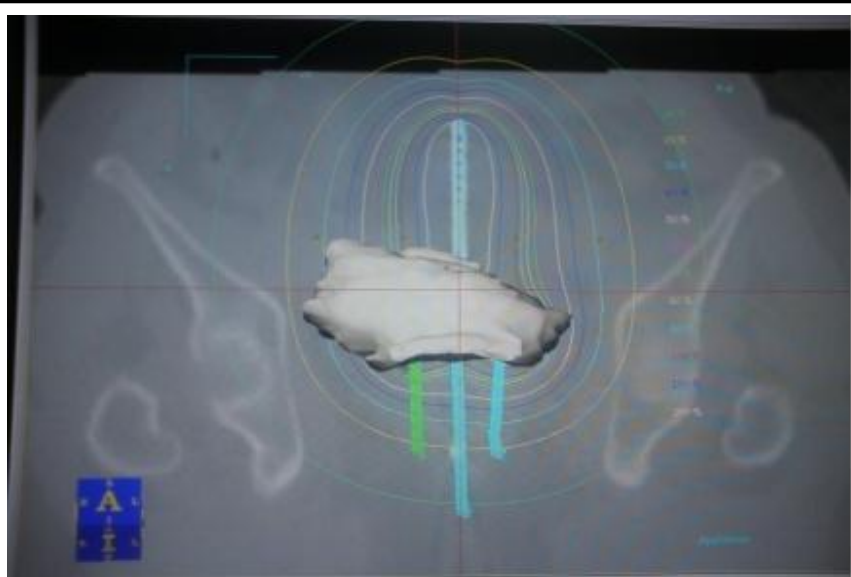

Figure 5 3D Reconstructed Images with Bladder Contoured and Iso Dose Distribution AP View

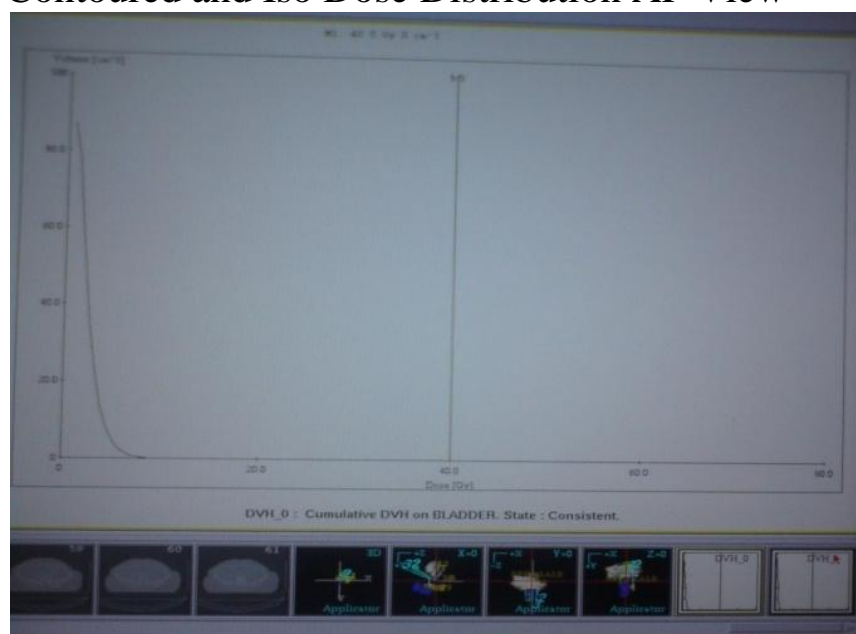

Figure 6 Cumulative Dose Volume Hisogram on Bladder

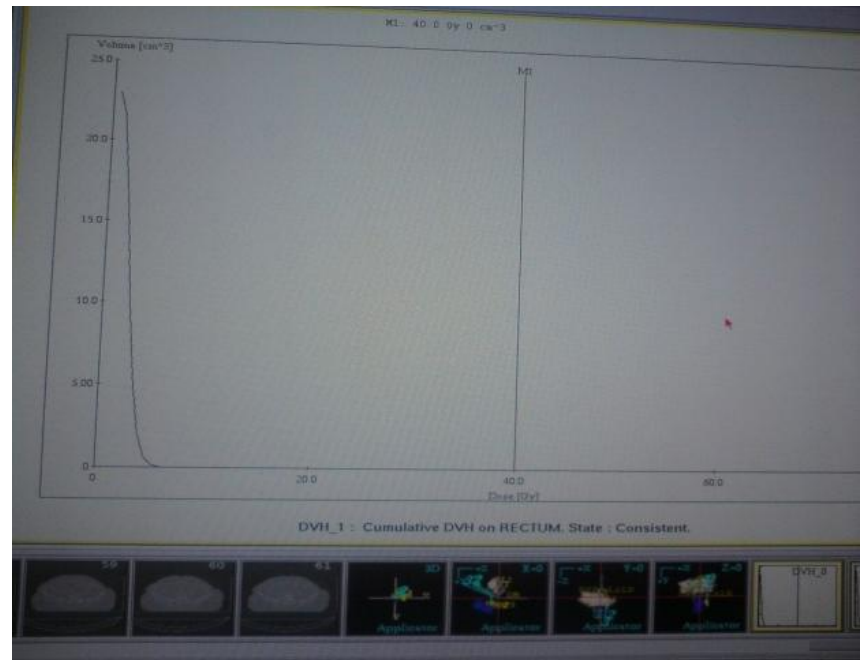

Figure 7 Cumulative Dose Volume Hisogram on Rectum

\section{Results}

From June 2010 to August 2010(ARM A) and from January 2016 to June 2016 (ARM B) Total of 30 cases of locally advanced cancer cervix patients were included in the study. 


\section{JMSCR Vol||07||Issue||11||Page 818-829||November}

Characteristics

1. Age

\begin{tabular}{|l|c|c|}
\hline Age (Year) & $\begin{array}{c}\text { Number of } \\
\text { cases(ARM A) }\end{array}$ & $\begin{array}{c}\text { Number of } \\
\text { cases(ARM B) }\end{array}$ \\
\hline $31-40$ & $3(10 \%)$ & 5 \\
\hline $41-50$ & $15(50 \%)$ & 12 \\
\hline $51-60$ & $12(40 \%)$ & 13 \\
\hline
\end{tabular}

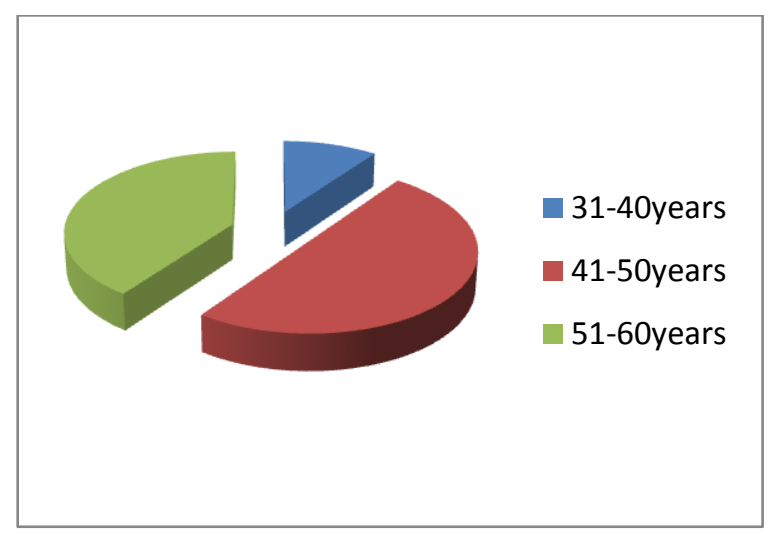

In this study we enrolled patients between 30-60 years of age. Majority of patients were distributed in the age group of 4 th and 5 th decades.

\begin{tabular}{|l|c|c|}
\hline $\begin{array}{l}\text { Stage } \\
\text { Grouping }\end{array}$ & $\begin{array}{c}\text { Number of } \\
\text { cases (ARM A) }\end{array}$ & $\begin{array}{c}\text { Number of cases } \\
\text { (ARM B) }\end{array}$ \\
\hline II B & 23 & 17 \\
\hline III A & 0 & 1 \\
\hline III B & 7 & 12 \\
\hline
\end{tabular}

Majority of the patients in the study belong to FIGO stage II B. Statistical analysis was performed by paired t-test comparing the doses of bladder and rectum in orthogonal $\mathrm{x}$-rays and CT.

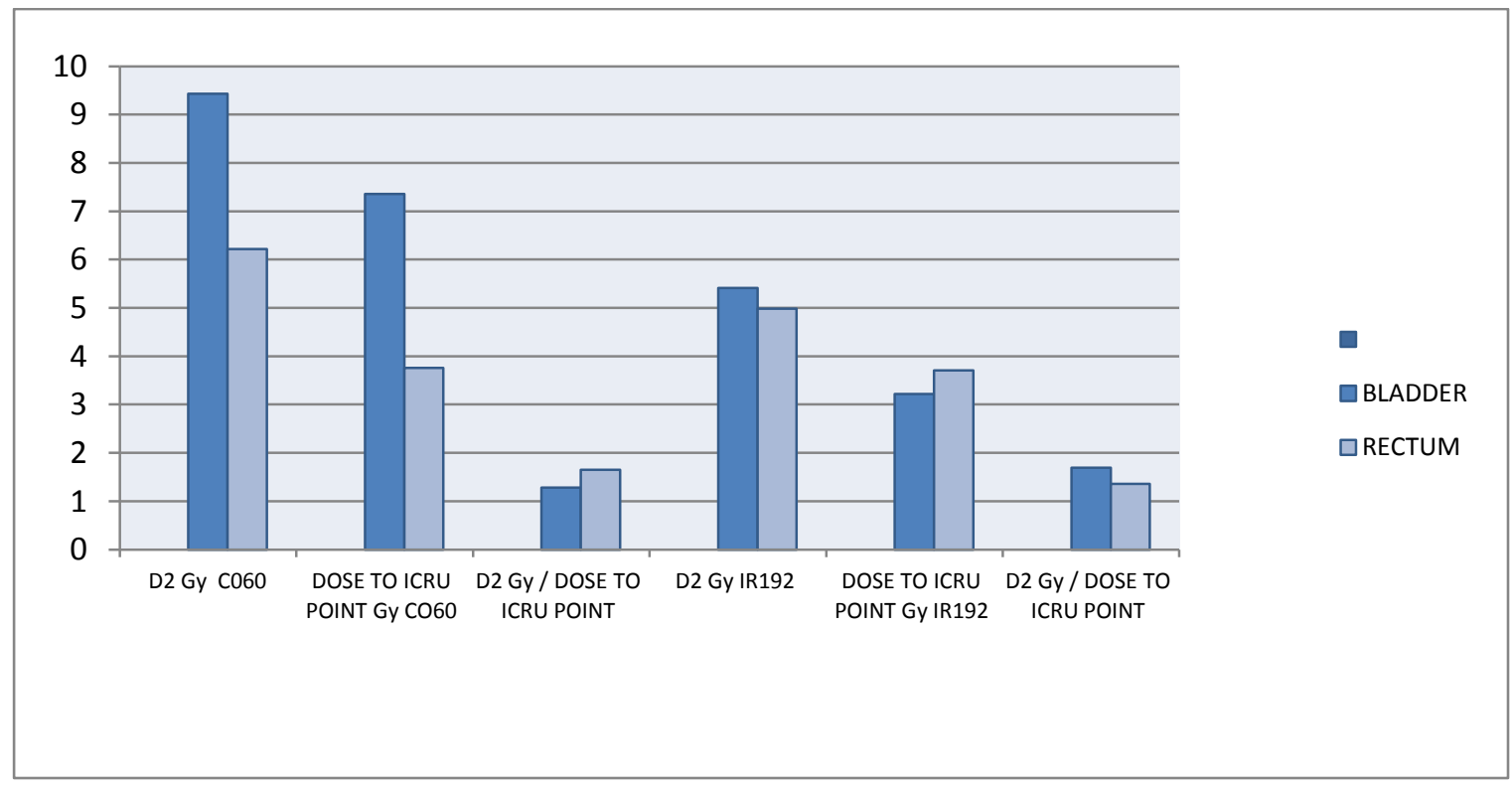

\begin{tabular}{|l|c|c|c|c|c|c|}
\hline ORGAN & $\begin{array}{c}\text { D2 Gy } \\
\text { CO60 }\end{array}$ & $\begin{array}{c}\text { DOSE TO ICRU } \\
\text { POINT Gy CO60 }\end{array}$ & $\begin{array}{c}\text { D2 Gy / DOSE } \\
\text { TO ICRU POINT }\end{array}$ & $\begin{array}{c}\text { D2 Gy } \\
\text { IR192 }\end{array}$ & $\begin{array}{c}\text { DOSE TO ICRU } \\
\text { POINT Gy IR192 }\end{array}$ & $\begin{array}{c}\text { D2 Gy / DOSE } \\
\text { TO ICRU POINT }\end{array}$ \\
\hline BLADDER & 9.43 & 7.36 & 1.28 & 5.41 & 3.22 & 1.69 \\
\hline RECTUM & 6.22 & 3.76 & 1.65 & 4.98 & 3.71 & 1.36 \\
\hline
\end{tabular}

The mean dose to high risk clinical target volumes (HRCTV) for D90 (dose to 90\% volume) was found to be $104.05 \%$ (SD):3.6. The mean D2cc (dose to 2 cubic centimetre volume) of the bladder, rectum were found to be17.9 Gy (SD: $0.56), 12.5$ Gy (SD: 0.92) and 4.1 Gy respectively. Fig. 3 shows the dose distribution in the frontal, sagittal and axial view. The detailed results of the study are given in Table 2. The dose to D2ccof the bladder, rectum by EBRT was $50 \mathrm{~Gy}$. The total dose by HDR brachytherapy and EBRT is given in Table 3. 


\section{Parametric Test}

To compare the means of two sets of scores, the ttest should be used. Since we were comparing the scores of the same respondents on two variables, the within-subjects (paired samples) t-test was used. The paired t-test involves taking the difference between the two scores for each respondents and finding the mean of these difference scores. The value of the t-statistics was shown, with its degrees of freedom (df) and its probability level (sig 2 tail). If the probability was less than 0.001, we concluded that there was a statistically significant difference between the means of the two sets of scores.

\section{Discussion}

\section{ARM A}

Dosimetry of ICA for carcinoma cervix was based on orthogonal radiographs with ICRU 38 recommendations, which allow the evaluation of point doses such as Manchester points A, B, ICRU rectal and bladder reference points. Orthogonal radiographs provide spatial

Information of the applicator with respect to bony structures. However, this time tested system has a limitation of computing the doses received by the volumes of the critical structures as this has only $2 \mathrm{D}$ value. There have been significant advances in imaging USG, CT, MRI. With an advantage of determine the dose volume parameters for the critical structures, We undertook this dosimetric study to compare, validate and document the correlation between point based doses to rectum and bladder with the conventional standard ICRU 38 rectal and bladder points. In the present study we demonstrated that more precise analysis on the dose received by certain volume of $(2 \mathrm{~cm} 3)$ of organ at risk, bladder and rectum can be accomplished by utilizing cumulative Dose Volume Histogram (CDHs) on CT plan, which may be of critical importance in regard to normal tissue tolerance limits. The dose to ICRU bladder reference point does not correlate with the maximum dose from the CT planning. Mean D2 was found to be $1.69+0.20$ times the mean ICRU bladder reference points. These results agree with the other studies published in the literature, where the ICRU bladder reference point underestimated the maximum dose by two to three times. The dose to ICRU rectal point from the radiograph based planning does not correlate with maximum dose from CT Planning. Mean D2 of rectum was found to be $1.36+0.25$ times the mean ICRU rectal reference point, suggesting that there was significant difference between the radiograph based ICRU rectal point and CT based estimation of the parameter D2.

The advent of better imaging modalities, radiation technology and its successful implementation in external beam radiation therapy is. Implementation of Various imaging modalities like ultrasound, CT have been explored in brachytherapy today. Imaging modalities have been invaluable in improving the quality of brachytherapy offered to cancer patients. Their introduction has led to improvements in treatment planning, implementation and assessment, resulting in efficacy and tolerability benefits for patients. CT based Brachytherapy planning is more or less standard of care . The advantages of CT based planning are accurate catheter reconstruction, better delineation of organ-at-risks (OAR) and documentation of dose volume parameters (DVH Parameters). However, the major disadvantage of inaccurate target volume delineation has paved way for MR based Brachytherapy.

\section{ARM B}

The results of this study are well in accordance with the ABS guidelines (80-90 Gy for D90HRCTV, 70-75 Gy for D2ccto the rectum and approximately 90 Gy for D2ccto the bladder.11-13The doses to the rectum, bladder are well within the tolerance limit without compromising the doses to target. Also the planning dosimetric parameters of this study concurred with the results of already published studies on Ir-192 HDR brachytherapy unit based planning. Bahadur et al.14has presented similar results with Ir-192 HDR brachytherapy in Ca Cx 
with target coverage of $100 \%$ to D90for HRCTV and D2cc of the rectum and bladder was $82.85 \%$ and 105.71 , respectively. The use of Co-60 HDR brachytherapy unit is comparable with the Ir-192 HDR brachytherapy unit. Palmer et al.7intheir study concluded that Co-60 may be used as an effective alternative to Ir-192 for HDR cervix brachytherapy, producing similar plans of equivalent D90, but with logistical benefits. There is a small dose increase along the extension of the source axis when using Co-60 compared to Ir-192, leading to small rectal dose increases for identical loading patterns. The treatment time by Co-60 HDR brachytherapy source is only 1.7 times more than that with Ir-192 HDR brachytherapy source with the initial activities.6The doses to organs at large distances from the applicators by Co-60 is the point of discussion compared to Ir-192, Venselaar et al.15.Co-60 source has higher dose values (ratio of 1.16 at $30 \mathrm{~cm}, 1.68$ at $45 \mathrm{~cm}$ and 2.57 at $60 \mathrm{~cm}$ ) suggesting higher integral dose for Co-60 sources. The study of Ntekim et al.16on acute gastrointestinal (GI) and genitourinary (GU) toxicity associated with Co-60 source in the brachytherapy of $\mathrm{Ca} \mathrm{Cx}$ shows that the median total biological effective dose (BED) for tumour was $86.2(84.4-88.8)$ while that for the rectum was 124.4 (120-133). $3 \%$ of patients had grade 3 gastrointestinal toxicity while all others had $\leq$ grade $2 \mathrm{GI}$ and GU toxicity and this is comparable with previous results. Therefore, Co-60 as HDR brachytherapy source is tolerable.

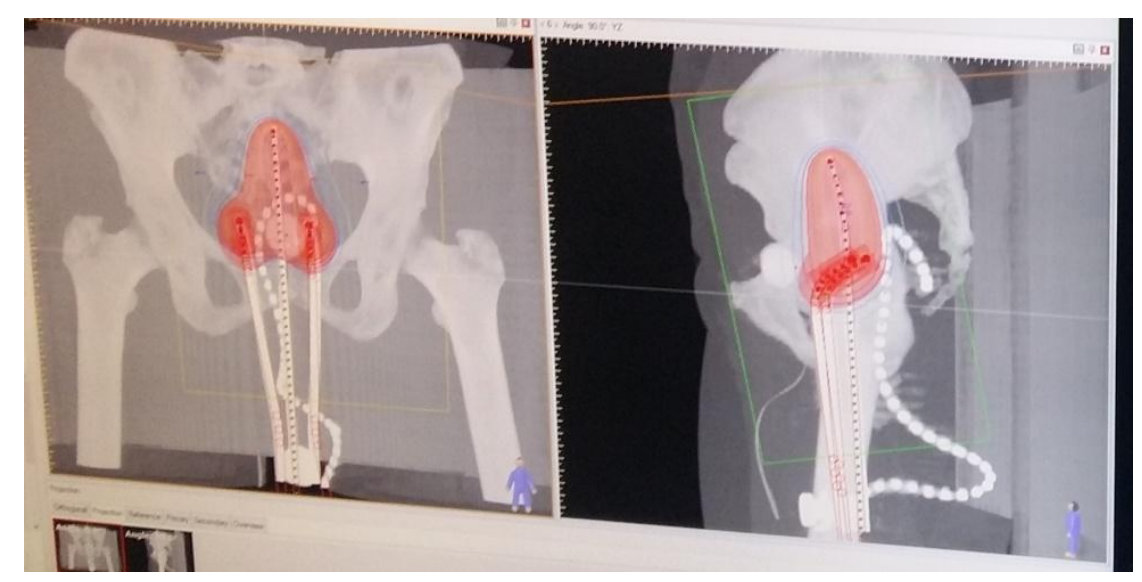

Fig 2a Coronal

Fig 2b Sagittal

Three channel Pear shaped dose distribution

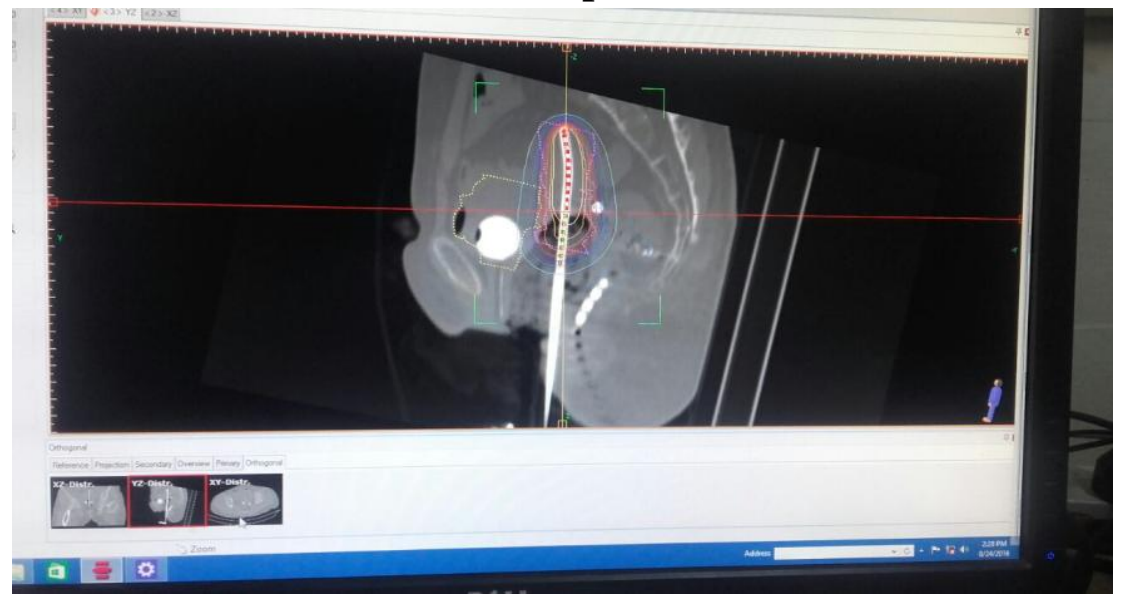

Fig 3 Dose distribution Sagittal view 


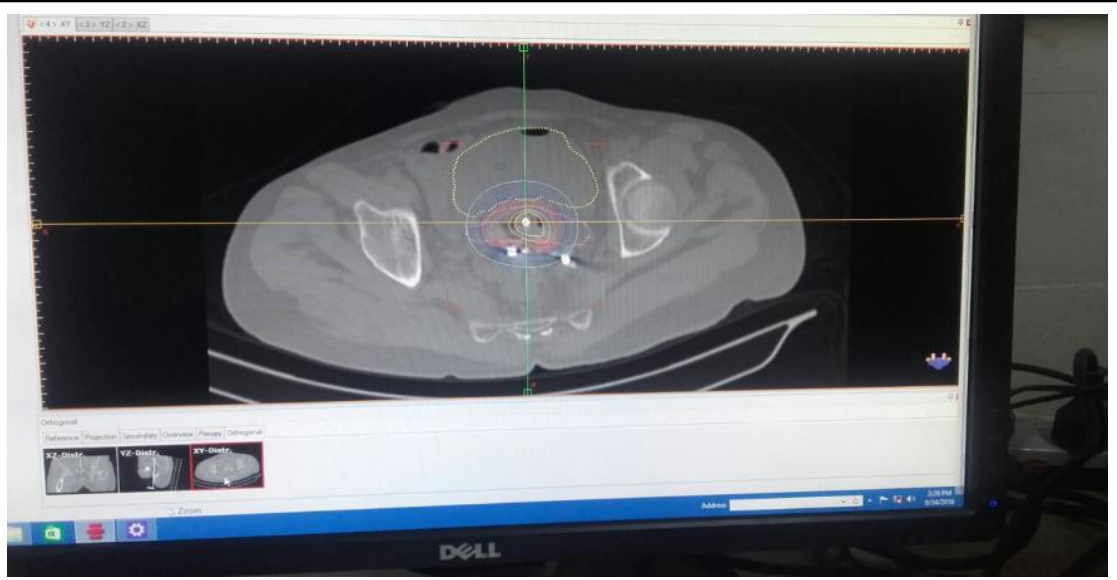

Fig 3 Dose distribution Axial view

\section{Conclusion}

CO60 HDR Brachythearpy is not producing bladder, Rectal complications and equally shows results on par with IR192. With long half life it is economical to run CO60 HDR Brachythearpy in high volume centres.

In all the plans of the Co-60 source based HDR brachytherapy of $\mathrm{Ca} \mathrm{Cx}$, with comparatively less target coverage and doses to OARs were achieved as per the ABS guidelines. Hence, it can be concluded that the Co-60 HDR brachytherapy unit is a good choice especially for the centres with large number of brachytherapy procedures as no frequent source replacement is required like in an Ir-192 HDR unit.

\section{Recommendation}

Target coverage was less than 80 Gy to Point A hence it was planned to change the fractionation from 8 Gy $/ 2$ \# to $7 \mathrm{~Gy} / 3 \#$.

From September 2016 institutional protocol was changed to $7 \mathrm{~Gy} / 3 \#$ and all patients in the study are under regular follow-up

Conflict of interest: None declared.

Financial disclosure: None declared.

\section{Acknowledgement}

\section{ARM A}

Radiation Oncology and Radiation physics Departments, Government Aringar Anna cancer hospital, Regional Cancer Centre, Kancheepuram

\section{ARM B}

Barnard Institute of Radiology and Oncology, Department of Radiotherapy and Radiation Physics, Madras Medical College, Chennai

\section{References}

1. Perez CA, Camel HM, Kuske RR, Kao MS, Galakatos A, Hederman MA, et al Radiation therapy alone in the treatment of Carcinoma of the uterine cervix: A 20 year experience. Gynecol Oncol 1986;23:12740. 1b. Carlos A. Perez, M.D., et al Radiation therapy morbidity in carcinoma of the uterine cervix: Dosimetric and clinical correlation Int. J. Radiation Oncology Biol. Phys., Vol. 44, 1999

2. Deshpande DD, Shrivastava SK, Pradhan AS, Viswanathan PS, Dinshaw KA. Dosimetry of Intracavitary applications in carcinoma of the cervix: Rectal dose analysis. Radi other Oncol 1997;42:163-6

3. Saarnak AE, Boersma M, van Bunningen BN, Wolterink R, Steggerda MJ. Interobserver variation in delineation of bladder and rectum contours for brachytherapy of cervical cancer. Radiother Oncol 2000;56:37-42.

4. Comparison between CT and Orthogonal Based Calculation of ICRU Rectal and Bladder Doses During Intracavity Brachytherapy for Cervix Cancer- Are Orthogonal Films now Obsolete? Alison Cameron1, Helen Coomber2, Chris 
French2, Paul Cornes1 -1Oncology Department, Bristol Haematology Oncology Centre (BHOC), UK; 2Radiotherapy Physics Unit, BHOC, UK, 2006.

5. Dosimetric evaluation of rectum and bladder using image-based CT planning and orthogonal radiographs with ICRU 38 recommendations in intracavitary brachytherapy Swamidas V. Jamema, Sherly Saju, Umesh Mahantshetty,1 S. Pallad,1 D. D. Deshpande, S. K. Shrivastava,1 and K. A. Dinshaw1. Department of Medical Physics, Tata Memorial Hospital, Mumbai, India. Department of Radiation Oncology, Tata Memorial Hospital, Mumbai, India 2007.

6. Schiffman M, Castle PE, Jeronim J, Rodrigue AC, Wacholde S.Human papillomavirus and cervical cancer. Lancet 2007;370:890-907.

7. Sankaranarayanan R, Ferlay J. Worldwide burden ofgynecological cancer: the size of the problem. Best Pract ResClin Obstet Gynaecol 2006;20:207-25.

8. Tyagi A, Supe SS, Kaushik S, Singh MP. A dosimetric analysis of $6 \mathrm{MV}$ versus 15 MV photon energy plans for intensitymodulated radiation therapy (IMRT) for carcinoma of cervix. Rep Pract Oncol Radiother 2010;15:125-31.

9. Banerjee R, Kamrava M. Brachytherapy in the treatment ofcervical cancer: a review. Int J Womens Health 2014;6:555-64.

10. Chandola RM, Tiwari S, Beck M, Chandrakar PK, Thakur SK.Experimental and Monte Carlo study of the effect of thepresence of dry air, cortical bone inhomogeneities and sourceposition on dose distribution of the mHDR-v2 source. J CancerRes Ther 2012;8:555-60.

11. Andrássy M, Niatsetsky Y, PérezCalatayud J. Co-60 versusIr-192 in HDR brachytherapy: scientific and technological comparison. Rev Fis Med 2012;13:125-30.
12. Palmer A, Hayman O, Muscat S. Treatment planning study ofthe 3D dosimetric differences between Co-60 and Ir-192sources in high dose rate (HDR) brachytherapy for cervixcancer. J Contemp Brachyther 2012;4:52-9.

13. Gurjar OP, Kaushik S, Mishra SP, Punia R. A study on roomdesign and radiation safety around room for Co-60 afterloading HDR brachytherapy unit converted from room forIr-192 after loading HDR brachytherapy unit. Int $J$ Health AlliedSci 2015;4:83-8

14. Strohmaier S, Zwierzchowski G. Comparison of60Co and192Irsources in HDR brachytherapy. J Contemp Brachyther 2011;3:199-208.

15. Khan FM. The physics of radiation therapy. 5th ed. Philadelphia:Lippincott Williams and Wilkins; 2014.

16. Dimopoulos JC, Lang S, Kirisits C, et al. Dose-volume histogram parameters and local tumor control in magnetic resonance image-guided cervical cancer brachytherapy. Int JRadiat Oncol Biol Phys 2009;75:56-63.

17. Dimopoulos JC, Potter R, Lang S, et al. Dose-effect relationship for local control of cervical cancer by magnetic resonance image-guided brachytherapy. Radiother Oncol2009;93:311-5.

18. Georg P, Lang S, Dimopoulos JC, et al. Dose-volume histogram parameters and late side effects in magnetic resonance image-guided adaptive cervical cancer brachytherapy. Int JRadiat Oncol Biol Phys 2011;79:356-62.

19. Bahadur YA, Constantinescu CT, Hassouna AH, EI-Sayed ME. Treatment planning for high dose rate brachytherapy of cervical cancer based on total dose constraints. Saudi Med J2011;32:495-503.

20. Venselaar JL, van der Giessen PH, Dries WJ. Measurement and calculation of the dose at large distances from brachytherapy 
sources: Cs-137, Ir-192 and Co-60. Med Phys 1996;23:537-43.

21. Ntekim A, Adenipekun A, Akinlade B, Campbell O. High Dose Rate Brachytherapy in the treatment of cervical cancer: preliminary experience with cobalt 60 radionuclide source $-\mathrm{a}$ prospective study. Clin Med Insights Oncol 2010;4:89-94. 